\title{
New Method for Insomnia Mongolian Mind-Body Interactive Psychotherapy in the Assessment of Chronic Insomnia: A Retrospective Study
}

\author{
Nagongbilige He $\cdot$ Wu Lan · Aruna Jiang $\cdot$ Haserden Jia \\ Shuzhi Bao · Longmei Bao - Altansha Qin · Orgel Bao • \\ Shinjiltu Bao · Nandin Wang · Suyaltu Bao · Shuangfu Dai • \\ Sarula Bao · Sarnai Arlud
}

Received: January 30, 2018 / Published online: June 19, 2018

(C) The Author(s) 2018

\begin{abstract}
Introduction: Insomnia is a common clinical complaint, and if not addressed it can increase the risk of developing other underlying diseases such as hypertension, depression and anxiety. The use of Mongolian mind-body interactive therapy as a comprehensive psychotherapeutic approach in chronic insomnia has been shown in this retrospective study.
\end{abstract}

Nagongbilige He and $\mathrm{Wu}$ Lan contributed equally to this manuscript.

Enhanced Digital Features To view enhanced digital features for this article go to https://doi.org/10.6084/ m9.figshare.6322958.

Electronic supplementary material The online version of this article (https://doi.org/10.1007/s12325018-0726-9) contains supplementary material, which is available to authorized users.

N. He $\cdot$ W. Lan $\cdot$ A. Jiang $\cdot$ S. Bao $\cdot$ L. Bao $\cdot$ A. Qin O. Bao $\cdot$ S. Bao $\cdot$ N. Wang $\cdot$ S. Bao $\cdot$ S. Dai $\cdot$ S. Bao S. Arlud $(\bowtie)$

Mongolian Psychosomatic Medicine Department, Inner Mongolia International Mongolian Hospital, Hohhot, Inner Mongolia, China

e-mail: sarinai@163.com

H. Jia

Mongolian Psychosomatic Medicine Department, Wu Hai Mongolian and Chinese Medicine Hospital, Wu Hai, Inner Mongolia, China
Methods: Subjects who had suffered insomnia for more than 1 month participated in the Mongolian mind-body interactive psychotherapy program between June 2012 and February 2014. They were interviewed by telephone at least 10 months before participating in the program. Their sleep was assessed using the Athens insomnia scale. Descriptive statistics, ANOVA and regression analysis were used for data analysis by SPSS software.

Results: Mongolian mind-body interactive psychotherapy significantly improved sleeping conditions. In ANOVA analysis, both short- and long-term outcomes were significantly affected by the treatment period. Patients who previously took medication and pre-treatment sleeping condition (ASI score) had a significant influence on long-term outcomes, as well as treatment time related to the duration of insomnia.

Conclusion: Mongolian mind-body interactive psychotherapy is a new method for insomnia, and narrative therapy and hypnotic methods together improve the sleeping condition, However, a further controlled randomized clinical study is needed to understand the efficacy.

Keywords: Chronic insomnia; Hypnosis; Mongolian mind-body interactive psychotherapy; Narrative therapy 


\section{INTRODUCTION}

Insomnia is a sleep disorder. People with insomnia have trouble sleeping, difficulty falling asleep, or staying asleep as long as desired [1]. It is common complaint both in the general population and in clinical practice. It may present the primary charge or in association with another physical or mental health problem. Pharmacologic therapies have proven effective in improving wake time after sleep onset and sleep maintenance, and in reducing the number of nighttime wakenings. Pharmacologic treatment has a high chance of producing side effects [2], but nonpharmacologic therapies could bring a long-lasting and reliable increase in the quality of sleep among people with chronic insomnia and have minimal side effects [3].

In China, patients have multiple choices for treatment of insomnia, including acupuncture [4-7], traditional medicine (Chinese, Mongolian, Tibetan traditional medicine) $[8,9]$ and concerns about western medicine [10], but psychotherapy is not very accessible, and even CBT-I isn't available in most hospitals. In Inner Mongolia, there is conventional Mongolian mind-body interactive psychotherapy (MMIP), doctors mainly use MMIP and traditional Mongolian medicine, and achieve better outcomes in multiple psychosomatic diseases than just using medication. MMIP is different from what is used by visiting psychiatric doctors or psychological therapists, it is practised like health education and people are free to come without thinking that the treatment is for "special people". MMIP can include both a large number of patients in group therapy (around 200-1000 people each time depending on the therapy room) and individual counselling. Group therapy uses case report narrative therapy and hypnotic methods together, and it is recommended that people attending group therapy begin with individual counselling. In MMIP, the patients' self-knowledge is important, and, through others' cases, they become aware of their own problems and are able to continuously deal with them.
In clinical practice, we have found all kinds of sleeping disorders have been improved or cured whatever the cause of the primary or secondary insomnia. Therefore, the aim of this retrospective study is to understand short- and long-term outcomes of MMIP for insomnia by using the Athens insomnia scale.

\section{METHODS}

\section{Data Source}

Our retrospective cohort study used the data period from June 2012 to February 2014 in the department of Mongolian psychosomatic medicine, Inner Mongolia International Mongolian Hospital, using patients who had participated in MMIP. The subjects self-reported insomnia lasting more than 1 month. They went through a two-step screening process that included an initial structured telephone interview followed by baseline data collection (Fig. 1).

\section{Compliance with Ethics Guidelines}

All procedures performed in studies involving human participants were in accordance with the ethical standards of the institutional and/or national research committee and with the 1964 Helsinki declaration and its later amendments or comparable ethical standards. Informed consent was obtained from all individual participants included in the study. The project ethical approval was obtained from the Inner Mongolia International Mongolian Hospital Ethics Committee.

\section{Therapy Details}

The therapy includes both large group therapy and individual counselling [11]. In group therapy, each session lasted $3 \mathrm{~h}$ and included 6-10 real cases of various diseases and symptoms, reporting how they dealt with their medical problems before and after MMIP, with an experienced doctor commenting and analyzing each case, which is an important part of the therapy. For more extensive applications, real 


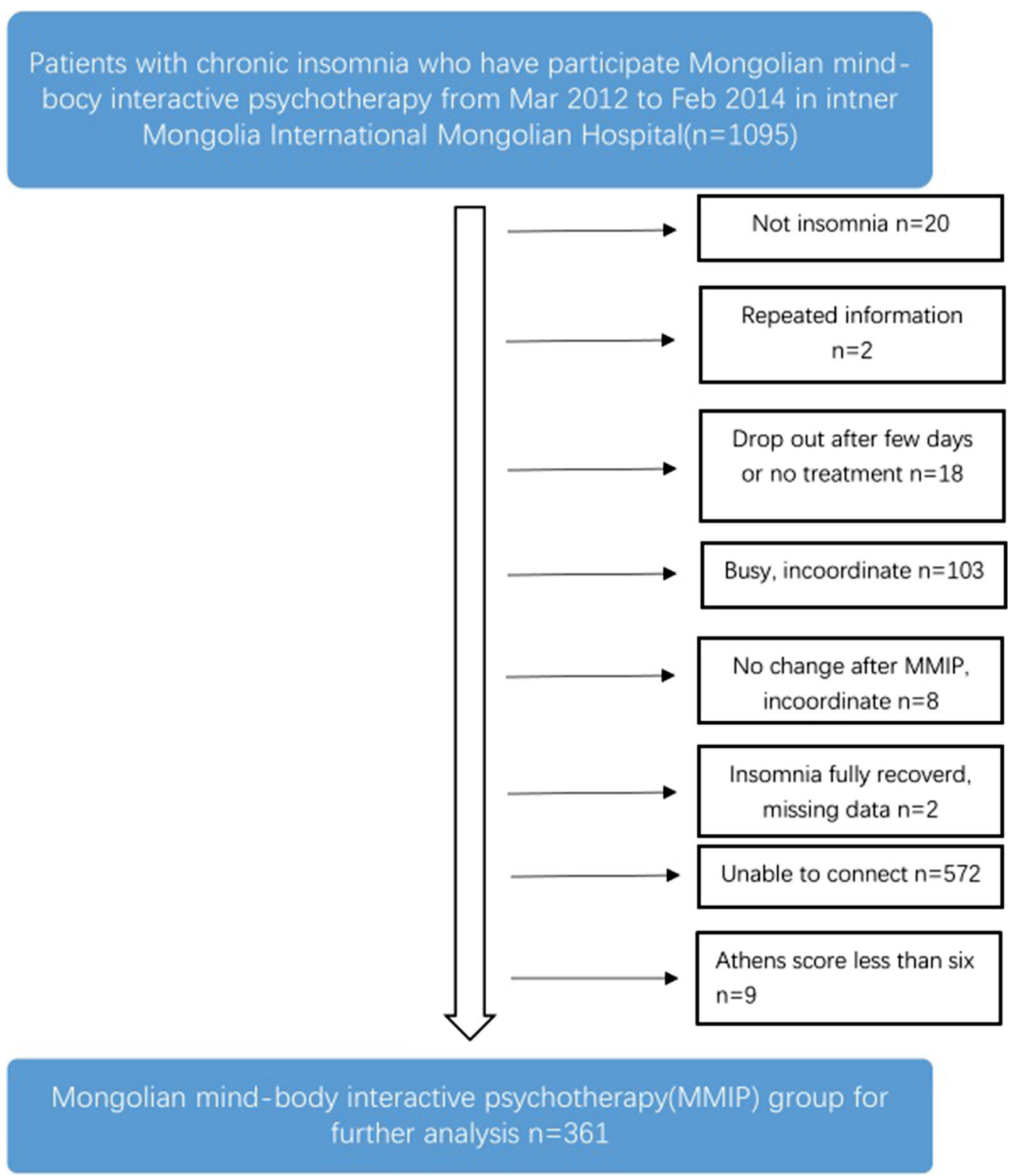

Fig. 1 Flowchart of subject recruitment at the department of Mongolian psychosomatic medicine of the Inner Mongolia International Mongolian Hospital

cases have been recorded and edited as video. We have presently stored around 4000 different real cases, and today there are 18 Mongolian hospitals practising video group therapy, the group number depending on the therapy room, involving about 200-1000 people each time. In the Inner Mongolia International Mongolian Hospital, the therapy room has around 400 seats, so most of the time around 400 people attend the video group therapy. The details of the video group therapy are: (1) at the start, four Mongolian songs will be performed for relaxation; (2) announcements for quiet and turning off of cell phones; (3) introduction of the treatment and the therapist; (4) the setting up of exercises, smoothly turning the neck, arm, hand, leg and foot; (5) the explanation of 6-10 different real cases (narrative psychotherapy is a core part of the therapy); and finally, (6) one more Mongolian song. Individual counselling 
does not investigate the patient's past or wrong behavior or cognition, only asking how they feel after MMIP and encouraging them to stay with MMIP and, when necessary, receive some Mongolian medication or other treatment.

\section{Telephone Interview}

A 20- to 30-min structured telephone interview was conducted to ensure that the participants' Athens insomnia scale (AIS) score is higher than 6 (i.e., symptoms consistent with insomnia) and that insomnia has continued for more than 1 month to be included in the study, but excluding cancer, depression and anxiety. The participants' sleeping condition pre-treatment, post-treatment and long-term (>10 months later), are established, using the AIS. Baseline information and previous medication information are included.

\section{Athens Insomnia Scale}

This scale assesses the severity of insomnia using diagnostic criteria set forth by the International Classification of Diseases (ICD-10). The eight-item questionnaire evaluates sleep induction, wakenings during the night, final awakening earlier than desired, total sleep duration, overall quality of sleep, sense of well-being during the day, functioning, and sleepiness during the day [12]. The study suggests that a cutoff score of 6 correctly distinguishes between insomnia patients and controls in $90 \%$ of cases.

\section{Statistical Analysis}

All data were analyzed using the IBM SPSS v.22 (IBM, Armonk, NY, USA). A review of sociodemographic data, medical records, and medication was conducted. AIS score was evaluated with paired two-tailed $t$ tests. The eight items of AIS were compared using nonparametric Wilcoxon tests. Short-term outcome $=$ (pre-treatment AIS score - post-treatment AIS score)/pretreatment AIS score, and long-term outcome $=($ pre-treatment AIS score $->10$ months AIS score)/pre-treatment AIS score. ANOVA analysis utilized for factors might affect the outcomes. Regression analysis fwas used to understand the relationship between treatment time and the duration of insomnia.

\section{RESULTS}

A telephone survey identified 1095 chronic insomnia residents who had participated in the Mongolian mind-body interactive therapy in March 2012 to Febriary 2014. Of these, 572 had changed their phone number or could not be contacted, 111 were not available (103 because they were too busy and 8 complained about the invalidity of MMIP). A total of 370 were enrolled, but 9 were excluded because their AIS score was $\leq 6$, leaving 361 for further analysis. The mean age of the 361 participants was $49.38 \pm 12.19$ years, $74.5 \%$ were women, $97.2 \%$ Chinese-Mongolian, the rest Chinese-Han. Regarding treatment for insomnia, 8.9\% had used diazepam, $15.2 \%$ had used Mongolian prescription medicine, $64 \%$ did not use any treatment, and $0.8 \%$ had tried multiple therapies. The mean duration for insomnia was $7.14 \pm 6.09$ years. The mean Mongolian mind-body interactive therapy treatment period was $28.32 \pm 21.98$ days (Table 1 ). Self-reported reasons for insomnia are given in supplementary Table 1.

The AIS score of post-treatment $(4.30 \pm 4.82)$ and $>10$ months later $(6.04 \pm 5.66)$ were significantly changed compared to pre-treatment $(15.05 \pm 4.57)$ using paired $t$ tests $(p<0.001)$ (Table 2$)$. Comparing before, after, and 10 months later, all eight items (sleep induction, wakenings during the night, final wakening earlier than desired, total sleep duration, overall quality of sleep, sense of well-being during the day, functioning, and sleepiness during the day) showed significant improvements $\quad(p<0.001) \quad$ (supplementary Table 2). AIS score cutoff at 6 distinguishes $90 \%$ of insomniacs from normal people,and in our study, post-treatment and > 10 months, insomnia had improved to normal $70.9 \%$ and $60.7 \%$, respectively.

In ANOVA analysis, both short- and longterm outcome are significantly affected by treatment period $(F=1.798, \quad p=0.001$ and 
Table 1 Characteristics of patients

\begin{tabular}{ll}
\hline Baseline data & \\
\hline Gender & \\
Female & $(n=274) 74.5 \%$ \\
Male & $(n=96) 25.5 \%$ \\
Nation & \\
Mongolian & $97.2 \%(n=360)$ \\
Chinese & $2.8 \%(n=10)$ \\
Age $(M \pm$ SD) (range $)$ & $49.39 \pm 12.19$ years \\
& $(13-88$ years $)$ \\
Family history & \\
Have & $5.8 \%(n=21)$ \\
Not & $45.4 \%(n=164)$ \\
Missing data & $48.8 \%(n=176)$ \\
Previously took medication & \\
Missing data & $1.4 \%(n=5)$ \\
No treatment & $6.14 \pm 6.09$ years \\
Diazepam $(1-4$ tablets/day $)$ & $64.0 \%(n=23)$ \\
Occasionally diazepam & \\
Mongolian medicine & \\
Chinese medicine & \\
Multiple treatment & \\
\hline
\end{tabular}

$F=1.683, p=0.003$, respectively) and those who previously took medication $(F=2.819$, $p=0.011$ and $F=2.675, p=0.015$ ) and those with pre-treatment sleeping condition (ASIscore) have a significantly influenced long-term outcome $(F=1.891, p=0.016)$. Sex, family history and reasons for insomnia did not have a significant impact on any outcome (Table 3).

Through regression analysis, treatment time was strongly related to the duration of insomnia $(B=0.626, t=3.212, p=0.001)$.

\section{DISCUSSION}

Mongolian mind-body interactive psychotherapy (MMIP) is a comprehensive therapy. It includes video group therapy (every day), group therapy (average once per month) and individual therapy (rarely). MMIP has no limitations concerning treatment sessions, it depends on the patient's own will. In MMIP group therapy, narrative methods are combined with hypnosis. In real cases, usually using examples of severe cases or chronic diseases with patients who have improved remarkably or been cured entirely (like cancer, leukemia, psoriasis, paralysis, brain tumor, insomnia, hypertension, fatty liver, enteritis, slipped discs, autism, depression, and so on), and therapy combined with hypnotic methods through relaxing in a quiet and dark environment, MMIP first gives people confidence, and then the participants will learn from others' false thoughts and behaviors, with selfknowledge coming from these cases of others. In MMIP, both psychological and physical stress reduction is the main task. When dealing with insomnia, cognitive behavior therapy is mostly

Table 2 Comparisons of sleep condition with AIS-8 total score

\begin{tabular}{llll}
\hline Athens score & Pre-treatment & Post-treatment & $>$ 10 months \\
\hline Mean $(\mathrm{SD})$ & $15.05 \pm 4.57$ & $4.30 \pm 4.82^{* * *}$ & $6.04 \pm 5.66^{* * *}$ \\
$t$ value & & 33.51 & 27.07 \\
Athens score range & $7-25$ & $0-24$ & $0-24$ \\
Athens score $\geq 6$ & $0 \%$ & $70.9 \%$ & $60.7 \%$ \\
\hline
\end{tabular}

Compared to pre-treatment AIS-8 score, the difference is significant using $t$ tests $\left({ }^{* * *} p<0.001\right)$ 
Table 3 Analysis of ANOVA

\begin{tabular}{|c|c|c|c|c|}
\hline & \multicolumn{2}{|c|}{ Short-term outcome } & \multicolumn{2}{|c|}{ Long-term outcome } \\
\hline & $F$ value & $p$ & $F$ value & $p$ \\
\hline Sex & 1.159 & 0.282 & 3.591 & 0.059 \\
\hline Duration of insomnia & 1.146 & 0.294 & 1.266 & 0.188 \\
\hline Treatment period & $1.798^{* *}$ & 0.001 & $1.683^{* *}$ & 0.003 \\
\hline Family history & 0.196 & 0.659 & 0.029 & 0.865 \\
\hline Previous took medication & $2.819^{*}$ & 0.011 & $2.675^{*}$ & 0.015 \\
\hline Reason for insomnia & 0.955 & 0.496 & 0.781 & 0.604 \\
\hline Pre-treatment AIS-score & 2.383 & 0.001 & $1.891^{*}$ & 0.016 \\
\hline
\end{tabular}

Short-term outcome $=($ pre-treatment AIS score - post-treatment AIS score $) /$ pre-treatment AIS score; long-term outcome $=($ pre-treatment AIS score $->10$ months AIS score $) /$ pre-treatment AIS score

${ }^{*} p<0.05,{ }^{* *} p<0.01$

about offering education about sleep in order to target dysfunctional beliefs/attitudes about sleep. Cognitive therapists will directly question the logical basis of these dysfunctional beliefs in order to point out their flaws. If applicable, the therapist will arrange a situation for the individual to test these flawed beliefs [13]. In addition to this, MMIP targeting multiple diseases and symptoms together not only improves the sleeping condition but also the lack of clinical study and publications. However, CBT-I has been well addressed by numbers of studies and found to be an active form of treatment for insomnia [14]. CBT-I is also useful in the treatment of insomnia related to or caused by mood disorders, pain, alcohol-related disorders, arthritis or coronary artery disease [15-21]. Those who suffer from PTSD have also shown improvementa [15].

In MMIP, patients appear to have various "reacting symptoms" which are adverse reactions like, for example: sleepiness, vomiting, diarrhea, olfactory hallucinations, seeing lights, feeling worms crawling on the skin, pain, sweating, crying, and fever, most of them appearing to be aggravated during therapy. However, the symptoms can disappear by themselves and there are subsequently better feelings, for instance, when there are vomiting symptoms, the people have no discomfort in the stomach and no asthenia after vomiting, but feel much fresher than before. In CBT-I or hypnosis, there are no reports of "reaction symptoms".

In the first few weeks of treatment in CBT-I, there is often an acute reduction in total sleep time that can lead to the side effect of increased daytime sleepiness. Beyond that, improvements from CBT-I are typically not seen until 3-4 weeks into treatment [22], while a few research studies have examined the efficacy of nurse-led CBT-I in primary care settings [23]. However, in MMIP, there is a different strategy without sleep restriction, although for those who do not believe in this psychotherapy, it is more easy to drop out. Although CBT-I refers to individuals with specialized training, MMIP mainly uses narrative therapy which is difficult to train for and doctor needs to be highly experienced. This is why video psychotherapy has been developed.

Regarding outcome measures for insomnia, it has been found that there are more substantial effects of treatment on subjective measures such as questionnaires than on objective measures of actigraphy and polysomnography. However, this does not limit the meaning of the findings, since the diagnosis of insomnia relies on patient reporting and not on a diagnostic laboratory test [24]. From the results showed the duration of insomnia, previously taken medication had impacts on both short- and long- 
term outcomes $(p=0.003$ and $p=0.015$, respectively). However, the duration of insomnia is related to treatment time, and longer periods of insomnia duration require longer treatment times.

\section{Study Limitations}

This is a retrospective study with limited and incomplete information of the subjects. More importantly, in this study, no control group is compared with MMIP so the curative effect is uncertain. Also, the reasons for insomnia are self-reported data by patients, so the correction of it is unsure, and this might bring analysis bias. For previously taken medication, the medication name is usually unclear, especially for traditional prescription medication (Chinese and Mongolian medication).

\section{CONCLUSION}

In this study, MMIP as a method targeting stress reduction has significantly improved chronic insomnia; however, there still needs to be a randomized control study to further understand the therapeutic effect.

\section{ACKNOWLEDGEMENTS}

Funding. The study, the article processing charges, and the Open Access fee were supported by the Fundamental Research Funds of Inner Mongolia International Mongolian Hospital (no. GJMY-201504); the health and family planning commission of the Inner Mongolia autonomous region project (no. 201702012) and 2017 Inner Mongolia autonomous region science and technology innovation guide project: the evaluation of the therapeutic effect of Mongolian mind-body interactive psychotherapy on insomnia. All the authors have seen and approved the manuscript.
Authorship. All named authors meet the International Committee of Medical Journal Editors (ICMJE) criteria for authorship for this article, take responsibility for the integrity of the work as a whole, and have given their approval for this version to be published.

Author Contributions. Sarnai Arlud and Nagongbilige He were responsible for the data analysis and writing and submission paper, and Shuzhi Bao designed the study. Shuzhi Bao, Longmei Bao, Altansha Qin, Aruna Jiang, Suyaltu Bao, Shinjiltu Bao, Haserden, Orgel Bao, Shuangfu Dai, Nandin Wang, Wu Lan, Sarula Bao were responsible for telephone interviewed patients. Nagongbilige He created MMIP and responsible for therapy.

Disclosures. The authors (Nagongbilige He, Wu Lan, Aruna Jiang, Haserden Jia, Shuzhi Bao, Longmei Bao, Altansha Qin, Orgel Bao, Shinjiltu Bao, Nandin Wang, Suyaltu Bao, Shuangfu Dai, Sarula Bao and Sarnai Arlud) have nothing to disclose.

Compliance with Ethics Guidelines. All procedures performed in studies involving human participants were in accordance with the ethical standards of the institutional and/or national research committee and with the 1964 Helsinki declaration and its later amendments or comparable ethical standards. Informed consent was obtained from all individual participants included in the study. The project ethical approval was obtained from the Inner Mongolia International Mongolian Hospital Ethics Committee.

Open Access. This article is distributed under the terms of the Creative Commons Attribution-NonCommercial 4.0 International License (http://creativecommons.org/licenses/ by-nc/4.0/), which permits any noncommercial use, distribution, and reproduction in any medium, provided you give appropriate credit to the original author(s) and the source, provide a link to the Creative Commons license, and indicate if changes were made. 


\section{REFERENCES}

1. Merrigan JM, Buysse DJ, Bird JC, et al. Insomnia. JAMA. 2013;309(7):733.

2. Benca RM. Diagnosis and treatment of chronic insomnia: a review. Psychiatr Serv. 2005;56(3):332-43.

3. Edinger JD, Wohlgemuth WK, Radtke RA, et al. Cognitive behavioral therapy for treatment of chronic primary insomnia: a randomized controlled trial. JAMA. 2001;285(14):1856-64.

4. Cao H, Pan X, Li H, et al. Acupuncture for treatment of insomnia: a systematic review of randomized controlled trials. J Altern Complement Med. 2009;15(11):1171-86.

5. Zhang W, Huang Z, Jin Y. Acupuncture as a primary and independent treatment for a patient with chronic insomnia: one-year follow-up case report. Medicine (Baltimore). 2017;96(52):e9471.

6. Fu C, Zhao N, Liu Z, et al. Acupuncture improves peri-menopausal insomnia: a randomized controlled trial. Sleep. 2017;40(11):zsx153.

7. Chung KF, Yeung WF, Yu BY, et al. Acupuncture with or without combined auricular acupuncture for insomnia: a randomised, waitlist-controlled trial. Acupunct Med. 2018;36(1):2-13.

8. Zhou QH, Zhou XL, Xu MB, et al. Suanzaoren formulae for insomnia: updated clinical evidence and possible mechanisms. Front Pharmacol. 2018;9:76.

9. Yan X, Wang Y, Li X, et al. Wendan decoction for primary insomnia: protocol for a systematic review and meta-analysis. Medicine (Baltimore). 2017;96(47):e8906.

10. Owens JA. Pharmacology of sleep, in principles and practice of pediatric sleep medicine. New York: Elsevier Saunders; 2014. p. 53.

11. He $\mathrm{N}, \mathrm{He}$ A. Mongolian mind-body interactive therapy. Wuhan: Hubei Science \& Technology Press;2015.

12. Soldatos CR, Dikeos DG, Paparrigopoulos TJ. Athens insomnia scale: validation of an instrument based on ICD-10 criteria. J Psych Res. 2000;48(6):555-60.

13. Perlis ML, Smith MT, Benson-Jungquist C, et al. The Conceptual framework for CBT-I. Cognitive behavioral treatment of insomnia: a session-bysession guide. New York: Springer;2005;7-11.

14. Okajima I, Komada Y, Inoue Y. A meta-analysis on the treatment effectiveness of cognitive behavioral therapy for primary insomnia. Sleep Biol Rhythms. 2011;9(1):24-34.

15. Taylor DJ, Pruiksma KE. Cognitive and behavioural therapy for insomnia (CBT-I) in psychiatric populations: a systematic review. Int Rev Psychiatry. 2014;26(2):205-13.

16. Johnson JA, Rash JA, Campbell TS, et al. A systematic review and meta-analysis of randomized controlled trials of cognitive behavior therapy for insomnia (CBT-I) in cancer survivors. Sleep Med Rev. 2016;27:20-8.

17. Rybarczyk B, Mack L, Harris JH, et al. Testing two types of self-help CBT-I for insomnia in older adults with arthritis or coronary artery disease. Rehabil Psychol. 2011;56(4):257.

18. Vitiello MV, Rybarczyk B, Von Korff M, et al. Cognitive behavioral therapy for insomnia improves sleep and decreases pain in older adults with comorbid insomnia and osteoarthritis. J Clin Sleep Med. 2009;5(4):355.

19. Brooks AT, Wallen GR. Sleep disturbances in individuals with alcohol-related disorders: a review of cognitive-behavioral therapy for insomnia (CBT-I) and associated non-pharmacological therapies. Subst Abuse. 2014;8:55.

20. Martínez MP, Miró E, Sánchez AI, et al. Cognitivebehavioral therapy for insomnia and sleep hygiene in fibromyalgia: a randomized controlled trial. J Behav Med. 2014;37(4):683-97.

21. Peoples AR, Garland SN, Perlis ML, et al. Effects of cognitive behavioral therapy for insomnia and armodafinil on quality of life in cancer survivors: a randomized placebo-controlled trial. J Cancer Surviv. 2017;11(3):401-9.

22. Edinger JD, Means MK. Cognitive-behavioral therapy for primary insomnia. Clin Psychol Rev. 2005;25(5):539-58.

23. Edinger JD, Sampson WS. A primary care "friendly" cognitive behavioral insomnia therapy. Sleep. 2003;26(2):177-82.

24. Mitchell MD, Gehrman P, Perlis M, et al. Comparative effectiveness of cognitive behavioral therapy for insomnia: a systematic review. BMC Fam Pract. 2012;13:40. 